\title{
Risk Management and Monitoring Methods for the Future Mother, Embryo, Fetus, and post-natal Consequences
}

\author{
Evelyne Jacqz-Aigrain, ${ }^{1}$ Véronique Lamarque ${ }^{2}$ and the participants in Round Table $N^{\circ} 5$, Giens XXI \\ 1 Pharmacologie Pédiatrique et Pharmacogénétique, Hôpital Robert Debré, Paris, France \\ 2 Laboratoires Pfizer, Paris, France
}

\section{Keywords:}

risk management;

drugs;

pregnancy;

pharmacovigilance;

network

\begin{abstract}
Data required to asses the risk of a new drug regarding the normal course of pregnancy as well as embryo, fetal and neonate development, are often missing when a new product is launched. In such a situation, a risk management plan is to be developed by the industrial and validated by regulatory authorities.

This risk management plan is to take into account the data benefits on the drug and its potential therapeutic use by women as being of childbearing age. The obtaining of post licence human data is to be built on many players, both private and public, involved in the data collection and evaluation. The setting up of such a network would allow them to join together and optimize their action by standardizing the data collected and their follow up. This should help to generate or rapidly respond to an alert, to conduct collaborative pharmacovigilance pharmacology studies.
\end{abstract}

\section{Introduction}

Drugs taken during pregnancy can affect the normal course of pregnancy as well as embryo and fetal development. Clinical studies, and specifically drug trials, are difficult to conduct in pregnant women, and the requirements for assessing the benefit risk ratio of a drug are different from those, which characterize clinical studies and "usual" therapeutic trials, conducted outside pregnancy. Risk management plans, a new regulatory requirement, must be adapted to this special situation.

\subsection{Clinical situations in practice}

There are two situations where drug risk assessment for pregnant women, the embryo, fetus, neonate and infant may be necessary:

- an individual risk assessment may be necessary for a pregnant woman and her physician: the latter may be required to asses the risk of a drug that has already been taken, or the risk of a drug that he/she wants to prescribe. This situation raises the

^ For the list of participants, please see the end of the article. difficult issue for the practitioner of access to quality information and to extrapolate the population data to an individual situation;

- it can also be risk management assessment of drugs within a given population who may raise a public health issue and in this case the problem is very different.

Only the latter issue was discussed during this roundtable discussion.

\subsection{Drug consumption in pregnant women; epidemiologic data}

Much research ${ }^{[1-3]}$ has reported significant drug consumption in pregnant women. Since 2000, studies published in France have shown prescription rates ranging from $93.5 \%$ in the Loire with a mean of 10.9 drugs per woman including 3.5 during the first trimester, to $99 \%$ in Haute-Garonne, with a mean of 13.6 drugs per woman, including 5.2 in the first trimester.

This overall prescription rate is significantly higher than the one reported in other countries: 80 to $86 \%$ in the US, $85 \%$ in Germany, $68 \%$ in the UK or still $42 \%$ in Sweden. ${ }^{[4-7]}$ The differences are probably linked to differences in the management of 
pregnant women among the various countries. For instance, the use of venotonics largely used, by the entire French female population.

\section{Review and insight gained from previous roundtables}

Three previous round table discussions have afforded the opportunity to discuss certain key aspects of problems raised by exposure to a drug during pregnancy.

The contribution of toxicology studies in reproduction to risk assessment during pregnancy was discussed in 1998, the International Conference of Harmonisation was used as the basis for the work carried out. The outcomes of the roundtable focused on several areas: the need to standardize the performance and interpretation of these studies, their highly variable predictive value, important for teratogenesis studies, more limited for fetal or post natal toxicity. ${ }^{[8]}$

In 1997 , and then in $2002,{ }^{[9,10]}$ discussions were held on drug risk assessment methodologies in pregnant women and the methods in risk management. We will report here on the conclusions of this roundtable, notably as it pertains to pre-clinical, clinical data and their use.

These round tables provided for an initial approach to risk management in pregnant women. Subsequently, experimental teratology studies, with good predictive value of human risk, teratogeneous drugs shall not be introduced or kept on the market except for in exceptional cases and managed with a reinforced risk management plan. The absence of a teratogenic effect during preclinical studies is a very reassuring factor, since, to date, no drug assessed as being non teratogenic in animals studies (International Conference of Harmonisation, $\mathrm{ICH})^{[11]}$ has been proven to have a teratogenic effect in humans.

\section{New European regulations relative to drugs after market introduction}

The regulatory framework governing post marketing of drugs has been supplemented over these past years. In November 2004, ICH E2E guidelines ${ }^{[11]}$ were adopted in November 2004, defining the planning rules for drug safety monitoring activities in the period following market introduction. The identification of significant risks, reported during clinical development, or potential ones in addition to missing data, notably in unstudied subpopulations, enables to provide for specific actions based on these individual risks. The case of the pregnant woman is a key issue, since this population is generally excluded from clinical trials in the premarketing phase, resulting as a precaution in a contraindication in the summary of product characteristics.

The introduction of new pharmaceutical legislation in November $2005^{[12]}$ rounded out these guidelines with the concept of risk management: it includes in addition to recommended actions or activities, a risk minimisation needs assessment and plan preparation describing them with, if necessary, a communications plan, notably letters to healthcare professionals.

A risk minimization plan can entail different types of actions: prescription guides, patient leaflets, training programs, changes to drug prescribing and delivery requirements... These measures should also be the subject as an impact assessment in order to evaluate their efficacy and adapt an appropriate plan.

Subsequently, drugs for which significant risks have been reported or are predicted or for which key data lack must be the subject of risk management plans developed by the industrial and validated by regulatory authorities.

Among the identification and/or monitoring actions to implement, pharmaco-epidemiologic studies - mainly cohort studies, case control studies, registers hold a key place regardless of whether they are on site or conducted from a data base. This non interventional research, known as "observational studies", are studies for which the medical strategy intended for an individual lending him/herself to research is not set in advance by a protocol and results from standard practice as part of which the drug(s) are ordinarily prescribed, in compliance with the requirements set by the product license. No additional diagnostic or monitoring procedure must be required of patients.

A contraindication under the heading "Pregnancy" in the Summary of Product Characteristics, which is a frequent situation in this population, could in theory preclude these observational studies. Carrying out such studies is not authorized from a regulatory standpoint, even though they may be required as part of risk management plans. This apparent contradiction should be the subject of prior discussions with healthcare authorities, if this research is to be set up.

\section{Ethics and clinical trials in pregnant women}

Review of individual texts related to drug experimentation in pregnant women or the use of these same drugs after their market launch has enabled to achieve the following advances while also raise certain issues.

The law relative to public health care policy ${ }^{[13]}$ provides for in its article L1121-5 provisions for this population. Pregnant women, breast-feeding and (nursing) mothers may only 
be asked to participate in biomedical research in the following circumstances:

- either the importance of the desired benefit for themselves and/or for the child is such that it justifies the foreseeable risk run;

- or the research is justified in light of the desired benefit for other women who have the same condition or for their children, provided that research of the drug's comparable efficacy can be conducted in another category of the population. In this case, the predictable risks and requirements that the research entails must be of minimal nature;

- article 17 of the additional protocol to the human rights and biomedicine agreement related to biomedical research (European Council of 25 January 2005), defines minimal risk if, in light of the nature and scope of the procedure, it is possible expect that the research causes, at most, very little negative and temporary impact on the health of the individual in question. Similarly, a minimal constraint is characterized by the fact that it is possible to expect that adverse events may arise, which are at most temporary and very mild for the individual in question.

Indeed, it is possible both from an ethical and regulatory standpoint to conduct clinical trials in pregnant women providing:

- prior information on the potential risks and benefits for themselves, their pregnancy, their fetus, their future offspring and their fertility, should be given, orally and in writing, with if possible time given to think about it;

- research should be relevant for particular healthcare needs of a pregnant woman or her fetus, or for pregnant women in general;

- it should be founded on reliable experience in animals, mainly in terms of risks of teratogenecity and mutagenicity (international guidelines on ethics for biomedical research involving human beings).

\section{Clinical situations requiring risk monitoring}

Drug risk analysis in this situation must take into account two main types of factors:

\section{1. The data benefits on the drug}

- Therapeutic class and toxicologic risk profile of the drug and other drugs in the class already on the market;

- pre-clinical data available for the drug under review as stated above, experimental studies on teratogenesis have real predictive value of teratogenic effects in human species, while those on development toxicity are not (or only slightly) predictive of a fetal or neonatal toxic risk;
- use of data on human therapy, including notably the risks identified during therapeutic trials. Insofar as, data in pregnant women are generally lacking, data representing the same therapeutic class and used in pregnant women will be also taken into account.

\subsection{Potential therapeutic use by women as being of childbearing age}

Various factors needs to be analysed:

- the epidemiology of the disease to treat;

- the probability of pregnant women's exposure, depending especially of the therapeutic class, the place of the "new drug" in the management of the disease, the existence of alternative treatments, specifically the standard of care, older, known, for which data in pregnant women are available...;

- the type of maternal disease managed;

- acute disease, occurring during pregnancy, for which a short term treatment will be prescribed or occasionally used on an as needed OTC basis,

- chronic disease, known before the beginning of pregnancy, that may justify planning of pregnancy and possible treatment adjustments, for example, for an older, better known drug...

- the potential exposure of the pregnant woman by seminal fluid makes mutagenous risk assessment necessary, if the drug is used by a man before the onset of pregnancy.

\section{Obtaining of post license human data}

Only the post-licensing data on a certain number of pregnancies exposed enable to assess the risk of a drug among pregnant women and the collection of exposure data is therefore essential. $^{[14]}$

\subsection{Sources of information}

- Spontaneous reporting of an adverse event by health professionals to a Drug Safety Monitoring centre, a pharmaceutical company or a Regional Pharmacovigilance Centre. It entails the reporting of an adverse effect in the mother or new-born, having been exposed in utero. Spontaneous reporting of exposure, and not of an adverse effect are specific to pregnancy (and to a lesser degree paediatrics). They are often driven by a request for an assessment of potential risk, specifically a congenital abnormality. The data are often incomplete and there must be careful analysis of the causality relationship. 
Although these isolated descriptions are insufficient in the majority of cases for measuring the risk, they remain essential in providing a warning sign. Thus the repetition of identical descriptions for a molecule or a given therapeutic class can be sufficient for identifying an embryo-foetal risk and for taking the appropriate measures (for example NSAIDs [non steroidal inflammatory drugs] and renal and cardio-pulmonary toxicity, ACE [Angiotensin-converting enzyme] inhibitors or sartans and renal toxicity). Many examples illustrate the paths linked to the retrospective analysis of these data. Thus, an elevated risk of abnormalities, notably cardiac one complicating the maternal administration of lithium during pregnancy, had been suspected from the analysis of a document put together from spontaneous reporting. The conducting of a prospective study has afforded the opportunity to check that the initial risk had been overestimated. ${ }^{[15,16]}$ Specialised organisations currently in existence in many countries, inform professionals and patients on the risks of exposure during pregnancy and assure the prospective follow-up of information requests. They collect information on the progress and the end of pregnancy, and often the examination of the new-born with a follow-up via phone call. ${ }^{[17]}$ The studies undertaken are in the majority of cases collaborative and more and more frequently include a comparison group made up of patients exposed to nonteratogenic or other drugs, which have the same therapeutic use. The number of patients exposed is sometimes insufficient for the definitive conclusion of an absence of risk, nonetheless it is more about interesting first results when no other clinical data are available. The absence of an increased risk of abnormality or complication with relation to the expected level in a reference population or in a comparison group does not allow one to confirm the safety of the drug concerned, but it eliminates a large risk. Moreover, this type of study allows one to analyse parameters rarely taken into account, such as the risk of spontaneous miscarriage or the abortion rate.

- Active surveillance

Active surveillance enables to conduct epidemiologic studies, case-control studies or cohort studies. It is built on various tools:

- sentinel sites where systematic and exhaustive review of all patient cases and/or histories can be made. In pregnancy, these sentinel sites are the maternities where pregnancies exposed to a drug can be monitored prospectively;

- pregnancy registry, can involve malformations, drug exposure or a specific pathology. Several malformation registries exist in France: Center East registry was at the origin of proving the involvement of valproic acid in the occurrence of spina bifida. ${ }^{[18]}$
On an international level, the various registries created since the sixties have been consolidated into international organizations such as EUROCAT and the International Clearinghouse (ICBDMS). In both of these systems, the incidence of a few selected malformations is regularly calculated and simple statistical methods are applied to determine if significant changes have taken place. Indeed, the change in incidence of a rare malformation is likely to be detected. But that presupposes that eventual teratogenicity also has an effect as potent as thalidomide or isotretinoin.

The routine monitoring of the teratogenic effect of drugs is conducted through an unique method of data analysis for data collected through these registries (MADRE project MAlformation Drug Exposure surveillance of ICBDMS).

However, any association found to be significant between a drug and a malformation must be interpreted with precaution and tested in other case series then be used as a hypothesis for an ad hoc epidemiologic study. It is only after confirmation and confrontation that the teratogenic effect can be confirmed.

- Clinical research studies in pregnant women are essential to determine pharmacologic changes related to pregnancy and their implications in therapy. They involve the treatments for chronic maternal diseases, maintained throughout pregnancy. They can be pharmacokinetic trials designed to assess the changes in maternal evolution of a drug or its placenta transfer. ${ }^{[19]}$

It can be an actual therapeutic trial for the management of a pregnancy related disease. In these situations, the complexity of the protocol is related to the difficulty in selecting fetal benefit and risk parameters. Finally, pharmacogenetic studies must be conducted to identify genetic risk markers, notably fetal malformation. In this field, only large population studies will be able to bring together an adequate number of patients (parent-child pairs) needed for a retrospective pharmacogenetic analysis.

\subsection{Players involved at the national level}

Nationally, many players (both private and public) are involved at various levels in the collection, assessment of drugs in pregnant women and subsequently conveying information to both healthcare professionals and patients.

\subsubsection{Drug Safety monitoring structures}

a) National Pharmacovigilance network

There are 31 drug pharmacovigilance regional centers. Their mission is to collect, review, and ensure follow up of adverse events notified by practitioners in the region that they cover. 
In-fitting with the decree of 26 May 2005 on good pharmacovigilance practices, the centres must also record all information relating to the use of drugs at the time of pregnancy and assure the follow-up of these requests in order to document the outcome of pregnancy. An increasing number of pharmacovigilance regional centres record this information in a joint and specific computerised data bank, with these data possibly being used later on in collaborative studies [cf. 5.1] or to provide rapid information to Afssaps in the event of an emergency. Certain centres are also members of a European network of information services on teratogenic agents.

Some pharmacovigilance regional centers with an interest and recognition in the field of " pregnancy and pediatrics" could benefit from clear posting of their credentials in this field. The possibility and even the value of such a posting have been debated but have not been the subject of a consensus. These centers have developed an IT application (TERAPPEL) enabling to input and follow pregnancy observations.

b) The Center for "pregnancy" Information

- The Center for Information on Teratogenic agents (CRAT) informs on risks of diverse agents during pregnancy (drugs, radiation, virus, professional and environmental exposures...).

- Information on drugs administered during pregnancy and in children - IMAGE - It is an information unit on "Drugs and Pregnancy" whose main objective is to develop the information activity. Pregnant women are consulted upon request in the maternity.

\section{c) Anti-poison center}

Centers are probably less utilized than the pharmacovigilance regional centers. They have, however, useful information for review, mainly through the monitoring of requests on suicide related drug ingestion during pregnancy. Additionally, they have experience in the management and follow-up of requests from the general public.

d) Drug Safety monitoring departments in pharmaceutical companies

These departments seek to monitor drugs introduced onto the market by the firm to which they belong. They can inform practitioners who contact them and ensure the follow-up of observations. Multinational firms subsequently have a large data base. In general, firms only collate the observations that have been notified to them but they can also set up registries in one or several countries.
National drug safety monitoring data from the companies are sent to the French drug agency (Afssaps), just as are the data from the pharmacovigilance regional centers.

\section{e) Malformation registries}

There are 4 of them in France: Paris, Rhône-Alpes, Auvergne and Alsace. They record all cases of malformations in a given region, at birth or in fetus after an abortion. The collection of drug exposures is done retrospectively, at the time of recording the case, and it is limited to the first trimester of pregnancy. The use of data is made by each registry and in the international malformation study center of Rome, to which French centers contribute

\section{f) Neonatology/maternity networks}

An example is the AUDIPOG network. It has existed since 1994, and is made up of public and private maternities from all regions in France, using an obstetrical AUDIPOG file or not, computerized or not.

\subsubsection{Objectives and activities}

The network objectives, defined during its creation, were the following:

- monitor, year after year, perinatal health indicators, (recruitment indicators, practice indicators, and results indicators), through combining data from maternity files participating in the Network;

- provide to maternities, the assessment tool for their practices and results, by comparing analyses performed on their own files to those of the Sentinel Network;

- organize multicenter studies, at the request of one or several Network members.

These structures appear very differently on the objective and activity plans.

They were reviewed allowing identification of the following characteristics:

- type of question asked and data collection:

- exposures/prospective follow-up regarding the outcome of pregnancy and the status of the neonate,

- notifications of adverse events, regardless of type : malformation, toxic, fetal, neonatal effect. . .

- type of calls: local, regional, national...;

- computerization;

- advice/information via phone, mail;

- consultations, participation in medical staff meetings;

- combination of data, transmission... 
It seems that all the structures involved group together data obtained by spontaneous reports and/or solicited. They also fulfill a mission of information on "Drugs and Pregnancy" to physicians and/or direction to the public. The "Pregnancy" activity can be predominant, or even exclusive or is a part of a "non specialized" activity of drug safety monitoring. All centers ensure as extensively as possible the follow-up of observations to obtain the outcome of pregnancy. Nevertheless, the absence of direct contact with physicians and/or patients can, in certain centres, impede obtaining monitoring/follow-up data, and make their validation difficult.

\section{Recommendations}

\subsection{To be a risk management plan}

A risk management plan designed to achieve better drug monitoring after market introduction, is defined as a comprehensive and ongoing management process, if possible of risk minimization over the course of the drug life cycle in order to optimize their benefit/risk ratio.

It must satisfy optimally very different situations depending on the drug, its position in the therapeutic strategy outside of pregnancy and its toxicologic profile etc. It is not possible to provide for a "standard" risk management plan adapted to all drugs/all situations at risk. It is, however, possible to set objectives and guidelines designed to respond to different situations.

It is necessary to take into account the arguments related to pharmacology, experimental data, and toxic risks demonstrated in other populations (adult, enfant...).

It can therefore be minimally planned (simple data collection on exposure by drug safety monitoring systems in place) or, on the contrary, require optimized or even exhaustive follow-up. In both of these extreme cases, the response is optimization of existing system performance in terms of rapidity of response and transparency.

As discussed above, the structures involved are multiple and data are scattered, inaccessible or accessible later.

Mutualization and coordination of existing monitoring systems enable to satisfy simple objectives:

- monitor and analyze data better, notably analyze routinely initial exposures. Reporting detection statistical methods, in the process of assessment, can be used to make the assessment of spontaneous reports easier; ${ }^{[20,21]}$

- rapidly respond to an alert (rare situation in practice ... );

- anticipate or generate the alert (risk probability) and non alert (probability of non risk);

- circulate validated and standardized information to specialists.
This assumes an organization into a non-centralized, reactive network with a collaboration between the company and a drug safety structure.

\subsection{Organize a Mother Child Network}

Ideally, a structure that would enable to collect all drug (and toxic) exposures at each prenatal visit, by using a maternity book that would have the advantage of being prospective, and thus avoid bias related to the retrospective aspect of data. Such an organization was developed in Sweeden.

According to the French structure, the setting up of a MotherChild network needs the development of a Coordination Committee with the following objectives:

- joining together individual partners by including them in medical networks and facilities located in hospitals and clinics open to involved specialists: obstetricians, pharmacologists, industrials, to define objectives and setting up procedures;

- specify the relationship of the network with the national and European drug agency, relations with drug safety monitoring departments of pharmaceutical companies and public health insurances;

- standardize/align data collected about the mother, pregnancy, the fetus;

- define follow-up procedures of pregnancy, the parameters collected (specially on neonatality), the need for extended followup;

- develop an IT charter, enabling to standardize data collections, to develop quality control, to stipulate input timing;

- establish the network operating charter, and particularly define exchangeable data, specifically data accessibility, the conducting of ad hoc studies, accessibility requirements, submission to the French Drug Agency, Afssaps.

\subsubsection{Querying the network as part of a risk management plan}

Figure 1 illustrates the national mother-child network querying process when a risk management plan is set up.

- The network can be activated by the early detection of a signal on a drug. The analysis of all notifications of the network is conducted by a coordinating center by analyzing all the notifications (positive and negative ones) detected in the network and linked to the alert.

- The network can also be used by members to conduct a study using the network's data base. Study objectives, each's participation procedures are given by the study's scientific committee, coordinated by the main investigator and accepted by the network coordination committee. 


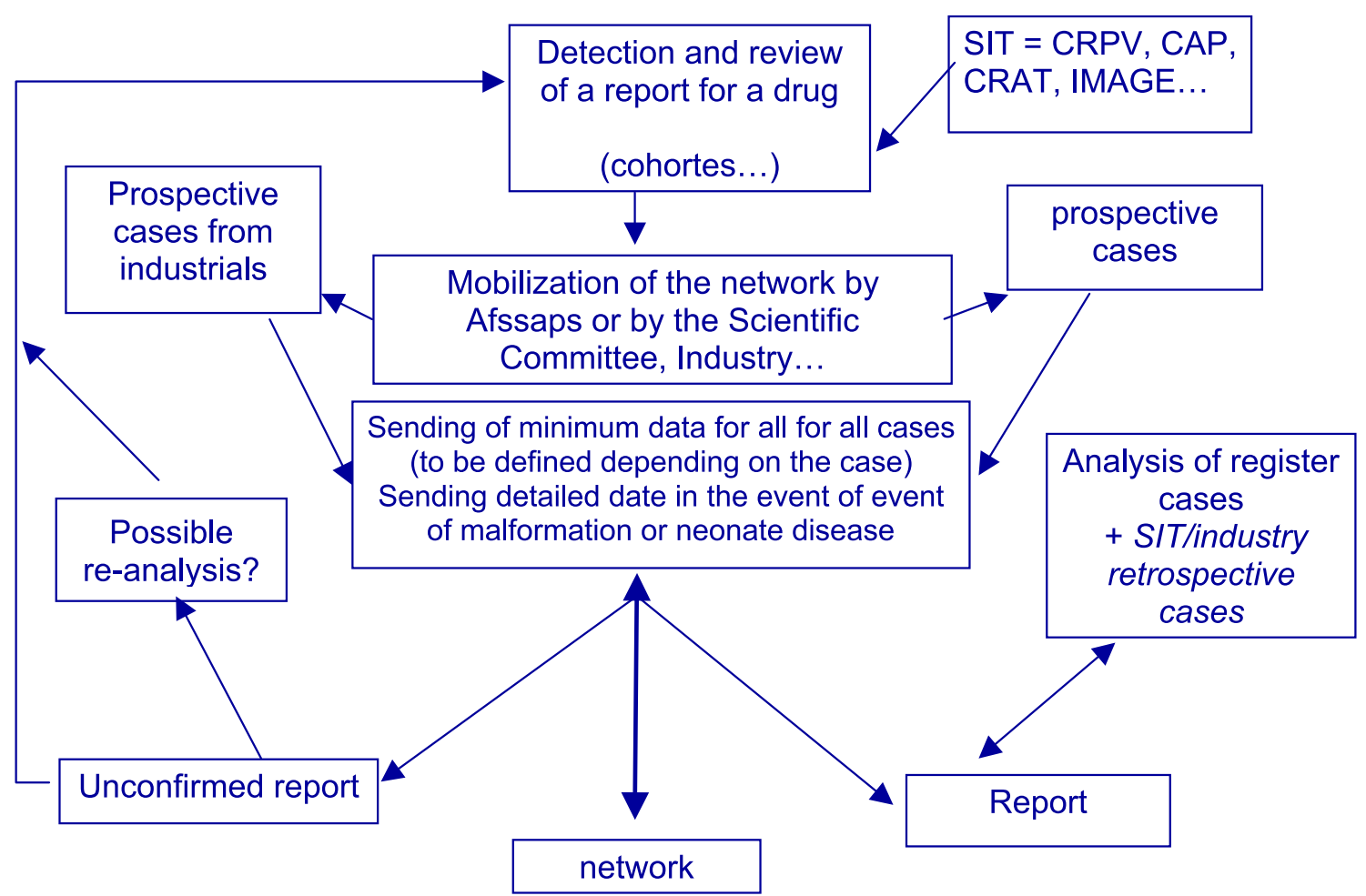

Fig. 1. What could be done quickly: make the mother-child network official. SIT: Services d'informations sur les tératogènes; CRPV: Centre Régional de Pharmacovigilance; CAP: Centre Anti-Poison; CRAT: Centre de Renseignements sur les Agents Tératogènes; Afssaps: Agence Française de Sécurité Sanitaire des Produits de Santé.

\subsection{The future of the Mother-Child Network}

Once organized, the mother-child network could meets tow major needs.

\subsubsection{Assistance in setting up and conducting collaborative drug safety monitoring studies}

Network partners from a broad range of horizons could:

- raise relevant questions;

- provide methodological aid (type of adapted study, choice of control populations... );

- organize, standardize, and conduct clinical research protocols, kinetic studies in pregnant or nursing women, neonates..., sample banks...

\subsubsection{Circulation of information to healthcare professionals and patients}

Currently, when scientific data are available, the information submitted by individual players may seem confusing, hesitant and result in inappropriate individual decisions. Assistance with individual risk management, whether it is random exposure, risk assessment of prescription or change in chronic therapy in view of a pregnancy must be a priority objective of the network. The stakes for information are key and the latter must be made consistent and provided by trained practitioners.

\section{Conclusion}

The set up of the risk management plans has become mandatory since 2005. Their application to pregnant women is particularly important in absence of any available human data. But varied clinical situations do not make it possible to roll out standardized plans. Nonetheless, it is almost always necessary to organize the follow-up of exposures or even run epidemiologic studies in collaboration with specialized structures. On the national level, many structures are involved in pregnancy and neonate monitoring. Their organization of the network would enable these structures to have all available information in a standardized format and also to be more reactive. 


\section{Participants}

E. Autret-Leca (Hôpital Bretonneau, Tours), L. Becquemont (CHU Bicêtre, Le Kremlin-Bicêtre), M.-J. Boutroy, (Maternité Régionale Universitaire, Nancy), P. Carlier (Hôpital Fernand Widal, Paris), A. Castot (Afssaps) C. Cornu (CIC Hôpital Louis Pradel, Bron), C. Damase-Michel (Faculté de Médecine, Toulouse), J.-P. Demarez (Pierre Fabre Médicaments), E. Dohin (Merck Sharpe \& Dohme Chibret), M. Gersberg (Sanofi-Aventis) C. Kreft-Jais (Afssaps) H. Le Louet (Hôpital Henri Mondor, Créteil) F. Meillier (LEEM), J.-L. Parier (Boehringer Ingelheim France), G. Pons (Hôpital Saint Vincent de Paul, Paris), D. Subtil (Hôpital Jeanne de Flandre, Lille), T. Vial (Hôpital Edouard Herriot, Lyon). We thank members of Round Table for their writting participation to this synthesis.

\section{References}

1. Beyens MN, Guy C, Ratrema M, et al. Prescription médicamenteuse pendant la grossesse en France : étude HIMAGE. Thérapie 2003; 58: 505-11

2. Felix RJ, Jones KL, Johnson KA, et al. Post marketing surveillance for drug safety in pregnancy: the organization of teratology information services project. Birth Defects Research 2004; 70: 944-7

3. Lacroix I, Damase-Michel C, Lapeyre-Mestre M, et al. Prescription of drugs during pregnancy in France. Lancet 2000; 356: 1735-6

4. Brocklebank JC, Ray WA, Federspiel CF, et al. Drug prescribing during pregnancy. A controlled study of Tennessee Medicaid recipients. Am J Obstet Gynecol 1978; 132: 235-44

5. Piper JM, Baum C, Kennedy DL. Pescription drug use before and during pregnancy in a Medicaid population. Am J Obstet Gynecol 1987; 157: 148-56

6. Rubin JD, Ferencz C, Loffredo C. Use of prescription and non-prescription drugs in pregnancy: the Baltimore-Washington Infant Study Group. J Clin Epidemiol 1993; 46: 581-9

7. Boethius G. Recording of drug prescriptions in the county of Jamtland, Sweden. II. Drug exposure of pregnant women in relation to course and outcome of pregnancy. Eur J Clin Pharmacol 1977; 12: 37-43
8. Guittin P, Soubrie C, Autret E, et al. Toxicologie de la reproduction : valeurs prédictives des modèles expérimentaux. Thérapie 1998; 53: 355-63

9. Elefant E, Sainte-Croix A. Evaluation du risque médicamenteux chez la femme enceinte : méthodologie d'evaluation et gestion du risque. Thérapie 1997; 52: $307-11$

10. Chauvenet M, Rimailho A, Hoog-Labouret N. Methodology for the evaluation of drugs in pregnant women. Thérapie 2003; 58: 247-58

11. Conférence Internationale d'Harmonisation, ICH. http://www.ich.org

12. Guideline on risk management systems for medicinal products for human use, EMEA/CHMP/96268/2005 http : //www . emea . eu . int

13. Loi 2004-806 du 9 août 2004 du Code de Santé Publique http://www.legifrance.gouv

14. Note for guidance on the exposure to medicinal products during pregnancy: need for post-authorisation data, EMEA/CHMP/1889/04 http: //www. emea.eu.int

15. Weinstein RM, Goldfield M. Cardiovascular malformations with lithium use during pregnancy. Am J Psychiatry 1975, 132: 529-31

16. Jacobson SJ, Jones K, Johnson K, et al. Prospective multicenter study of pregnancy after lithium exposure during first trimester. Lancet 1992; 339: 530-3

17. Clementi M, Di Gianantonio E, Ornoy A. Teratology information services in Europe and their contribution to the prevention of congenital anomalies. Community Genet 2002; 5: 8-12

18. Robert-Gnansia E. Méthodes d'évaluation des risques liés aux prises médicamenteuses pendant la grossesse. Avantages et limites de l'épidémiologie, interprétation des résultats. Thérapie 2002; 57: 175-80

19. Koren G. Clinical trials during pregnancy: Challenges and solutions. In Paediatric Clinical Pharmacology, p 359-365, 2005 edited by E. JacqzAigrain, I Choonara, Fontis Media Editors

20. Bates DW, Pappius EM, Kuperman GJ, et al. Medinfo. Measuring and improving quality using information systems 1998; 9: 814-8

21. Evans SJ, Waller PC, Davis S. Use of proportional reporting ratios (PRRs) for signal generation from spontaneous adverse drug reaction reports. Pharmacoepidemiol Drug Saf 2001; 10: 483-6

Correspondence and offprints: Evelyne Jacqz Aigrain, Service de Pharmacologie Pédiatrique et Pharmacogénétique, Hôpital Robert Debré, 48 boulevard Sérurier, 75019 Paris, France.

E-mail: evelyne.jacqz-aigrain@rdb.aphp.fr 